\title{
Assessing the influence of personal and organizational factors on surgeon's performance: a study on surgeons' perceptions
}

\author{
Rossella Onofrio ${ }^{1}$, Paolo Trucco ${ }^{1}$ \\ ${ }^{1}$ Department of Management, Economics and Industrial Engineering, Politecnico \\ di Milano, Via Lambruschini, 4/b 20156 Milano, Italy \\ \{rossella.onofrio, paolo.trucco\}@polimi.it
}

\begin{abstract}
In the emerging area of Human Reliability Analysis (HRA) applications in healthcare, a critical issue is the suitability or proper application of the existing taxonomies of Influencing Factors (IFs). The aim of the present study is twofold: i) providing surgeons' views about the IFs to be implemented in HRA applications to surgical procedures; ii) assessing surgeons' perception of the influence of personal and organizational factors on surgical performance in different surgical contexts (open and MIS surgery). The study methodology involved focus group and individual interviews for the former, a survey for the latter. Twenty IFs were identified as relevant for the surgical context, among a preliminary list of categories taken from extant literature. The difference of the perceived influence in the two surgical contexts, i.e. open vs laparoscopic, resulted significant for five Ifs: verbal interruptions; rude talk and disrespectful behaviors; unclear or failed communication; poor coordination; poor situation awareness.
\end{abstract}

Keywords: Human Factors · Performance Shaping Factors · Human Reliability Analysis

\section{Introduction}

A key question facing surgical units relates to the identification of factors that can influence surgical outcomes and, therefore, how knowledge of these factors can be used to enhance surgical performance. Research efforts across the world have sought to identify factors that may influence surgical performance, and with recent advances in surgical technology, a number of studies have focused on the association between surgical outcomes, surgical technology, and the technical skills and types of training of surgeons.

In the emerging field of Human Reliability Analysis (HRA) a critical issue in healthcare applications [1]-[4], as currently debated in literature, is the proper application or suitability of the existing taxonomies of Influencing Factors (IFs, also la- 
beled as Performance Shaping Factors or PSFs), since they were primarily designed and developed for industrial contexts.

Within HRA, the choice of PFs/IFs refers to the identification of personal or organizational factors that directly affect the human performance; their influence is generally assessed by means of experts' judgments and therefore is highly subjective. In healthcare applications, the choice of these factors becomes even more critical phase due to the high number of contingencies within a complex socio-technical environment: wide spectrum of patient conditions, variety of procedures, alternative technological settings and team configurations.

In the extant literature, many HRA methods modulate the Human Error Probability (or Unreliability) with a set of factors that may affect human performance. The terminology about context factors differs from one HRA technique to another. Some HRA techniques label these factors as "Performance Shaping Factors" (PSF) [5], "Performance Influencing Factors" (PIF), "Influencing Factors (IFs)" [6], or "Error Promoting Conditions (EPC)" [7] . In fact, several different taxonomies of contextual factors are proposed in industrial literature according to the different methods.

When it comes with the application of HRA in the healthcare context, at least three issues must be addressed for a proper integration of performance influencing factors.

Firstly, there is an open debate about which IFs should be used in HRA and what is the appropriate number of PSFs to be included in the analysis. In fact, "There is considerable range in the number of PSFs provided by individual HRA methods, ranging from single factor models such as time-reliability curves, up to 50 or more PSFs in some current HRA models.” [8]. For example: The US Nuclear Regulatory Commission advocates 15 PSFs in its HRA Good Practices [9], while its SPAR-H method [10] espouses the use of 8 PSFs and its ATHEANA method [11] features an open-ended number of PSFs. The apparent differences in the optimal number of PSFs can be partly explained referring to the level of detail of the human model or the different functions of PSFs in HRA [8] [12].

Secondly, PSFs are conceived in terms of the negative effects they might induce on human performance, even though a greater emphasis has been recently given to ways of modeling PSFs taking into account also their potential influence on enhancing human performance.

Thirdly, the terminology of the IFs is strictly connected to the meaning given in a specific context. For this reason, the assessment results among analysts could be different because they assess the factors with their subjective interpretations [12].

In healthcare there is need to design IFs taxonomy to be implemented in HRA for surgery, dealing with these HRA issues. Accordingly, the aim of the present study is twofold: i) providing surgeons' views about what are the IFs to be implemented in HRA applications to surgical procedures; ii) assessing surgeons' perception of the influence of personal and organizational factors on surgical performance. To this end, the following Research Questions are posed:

RQ1: "What are the IFs to be included into a taxonomy for HRA applications in surgery?"

RQ2: "What is the perceived influence of the IFs on surgeon's performance? Does the perceived strength of the influence vary under different surgical contexts (e.g. open vs laparoscopic)? 
The rest of the paper is organized as follows: section 2 provides the research method used; section 3 presents and discusses the main results; section 4 draws the conclusion and suggests directions for future research.

\section{Study Methodology}

The study builds on results of a previous study involving a literature review of influencing factors in surgery and an observational study [13]. The present study is divided into two parts, the first involving focus group and individual interviews undertaken with Danish and Italian surgeons and anesthetists, and the second a survey, undertaken with Italian surgeons.

\subsection{Qualitative study: Focus group and individual interview}

The design of IFS taxonomy items, to be implemented in the final taxonomy of IFs for HRA applications in surgery, was performed in Italy and Denmark between February and June 2015. We applied the focus group methodology [14], and individual semi structured interviews. This first phase of the study was aimed at capturing the ways in which surgeons conceptualize, cluster and articulate influencing factors.

In particular, the focus group held in Denmark (in March 2015) involved surgeons only $(\mathrm{n}=7)$, whereas the individual semi structured interviews involved both surgeons $(n=3)$ and anaesthetists $(n=9)$; in Italy semi structured interviews involved only surgeons $(n=5)$. The size of our focus group ( 7 participants) obeys to the common standard of four (minimum) to twelve (maximum) participants per group [14]. The participants were selected through consultation with the manager of the surgical department of the Hillerød Hospital. The informal nature of this methodology facilitated an interactive discussion and reflection about the surgeons' perception of factors that shape surgical performance.

We created an interview guide for both the focus group interview and individual semi structured interviews. It focuses on a brief presentation of the study and shows the participants an A4 paper including a table with the list of the macro categories of factors identified from a previous literature review study [13]. A set of IFs items was developed and turned into a checklist to gather data from surgeons about each factor category and assure the traceability of answers.

The discussion was primarily focused on labels, definitions, meanings of the factors to validate items and descriptors of influencing factors. To understand the relevant IFs items, when an interviewee was referring to the specific IFs item, the interviewers marked the checklist. When some IFs items were not mentioned, the interviewers asked additional questions about the missing ones. The ending of each interviews occurred when the participants had no further thoughts on IFs.

The focus group session lasted about one hour and half and was led by two moderators, who covered each of the IFs categories in the interview guide. Each of the individual semi structured interviews lasted around 30 minutes of discussion. All the interviews were audio-recorded and subsequently transcribed verbatim. Data analysis steps were discussed continuously among the authors, and when possible, with other scholars, ensuring the reliability of data analysis. 


\subsection{Survey method}

The results of the focus group and individual interviews in Italy and Denmark were used to design a questionnaire to assess surgeons' perceptions about the influence of IFs on surgical outcome. Survey method was chosen to elicit experts' judgments. The questionnaire was divided into three sections: the first one was dedicated to the instructions, the second one was dedicated to provide the perception about the influence of each of the IFs on their performance and the third one was dedicated to the collection of demographic information, in order to successively make comparisons among results. In detail, the question repeated for each of the twenty influencing factors (X) was:

"Please provide your subjective estimate of how often the factor X has had a negative influence on your own performance and the risk of adverse patient outcome in the operations in which you have participated during the last year?

Please indicate your estimate by a number from 0 and 10 where:

$0=$ the factor has had no influence at all, if present;

10 = the factors has had a negative influence in every operation, if present".

The demographic information we collected from surgeons were: the prevalent surgical technique used in the past year (with distinction among open, laparoscopic and robotic surgery); position/title of the surgeon; surgical speciality; years of experience; the surgical role during the last year of surgical activity, if in robotic surgery (i.e. first operator in console, or second operator at the operating table); number of surgical interventions performed (approximate number). Finally, two open questions were included; one about possible suggestion of additional factors not listed in the questionnaire, and a second one dedicated to other general comments, if any.

The questionnaire was designed in both paper and online versions. The software used to implement the online version of the questionnaire is "Typeform". Once the draft questionnaire has been designed we tested the questionnaire with a group of surgeons of the Urology Department of an Italian hospital, Ospedale Niguarda Ca' Granda in Milan.

A convenience sample of Italian surgeons, as members of the Italian Society of Surgery, was reached through emails, inviting them to answer the online questionnaire. The first email included a cover letter and a link to the questionnaire. The completion of the survey was estimated to take between 10 and 15 minutes. Two email reminders were sent to non-respondents.

Data was recorded in MS Excel, and subsequently statistical analysis has been done through the Statistical Package for the Social Sciences (Stata ${ }^{\circledR} 13$ ). 


\section{Results}

\subsection{Focus Group and individual interviews}

Twenty IFs resulted from the selection operated through the focus group and individual semi-structured interviews. Table 1 provides the list of IFs and respective descriptions.

\subsection{Survey results}

In this paper, we limited the description of the results to the descriptive statistics of the perceived influence of IFs on surgeons' performance. Firstly, we made a cumulative analysis of the entire sample, considering the surgical context as homogenous; secondly, we investigated if there is significant difference of surgeons' perception of the influence of IFs on their performance in the two surgical contexts under analysis (open and MIS surgery).

\subsubsection{Respondents characteristics}

A convenience sample of 93 surgeons, belonging to the Italian Society of Surgery, provided anonymous answers to the questionnaire administered online. Table 2 summarizes the demographic characteristics of the surveyed surgeons. Of the 93 responders, 56 are surgeons active in open surgery $(60 \%), 37$ predominantly operate in laparoscopic surgery $(40 \%)$.

\subsubsection{Descriptive profiles}

Figure 1 shows the cumulative descriptive profiles about the perceived influence of IFs considering the surgical context as homogeneous (93 respondents). The perceived influence of IFs with mean ratings $>=4$ concern: noisy use of social media (4.00); poor management of errors and threats to patient safety (4.06); rude talk and disrespectful behaviors (5.26); unclear or failed communication (4.04); lack of experience of surgical team colleagues (4.05); lack of experience of anesthetics team colleagues (4.47); fatigue (4.21); time pressure (4.48); poor use of technology (4.00). In Figure 1, the arrows indicate the IFs with the median $>=4.00$. In particular: noise and ambient talk $(\mathrm{mdn}=4.00)$; rude talk and disrespectful behaviors $(\mathrm{mdn}=5.00)$; improper use of procedure and checklists $(\mathrm{mdn}=4.00)$; unclear or failed communication $(\mathrm{mdn}=4.00)$; poor coordination (med=4.00); Lack of experience of surgical team colleagues $(\mathrm{mdn}=4.00)$; Lack of experience of anesthetics team colleagues $(\mathrm{mdn}=4.00)$; time pressure $(\mathrm{mdn}=5.00)$. 
Table 1. Validated list of IFs for HRA applications in surgery

1 Noise and ambient talk. Continuous or sudden noise; team members talking in the background or coming and going and moving around in a noisy way.

\begin{tabular}{ll}
2 & Music. Presence of background music in operating room. \\
\hline 3 & Noisy use of social media. Team members talking about and obtrusive-
\end{tabular} ly sharing social media content.

4 Verbal interruptions. Verbal Interruptions that are either untimely or not patient relevant.

5 Poor management of errors and threats to patient safety. Failure to share information promptly and openly about errors and threats to patient safety.

6 Poor guidelines, procedures or checklists. Guidelines, procedures or checklists are inadequate: lacking, too complex, or not at right level.

7 Rude talk and disrespectful behaviors. Derogatory remarks, behaviors showing lack of respect of OR team members, shouting and harsh tones of voice.

8 Improper use of procedures and checklists. The improper use, or non-use, of the WHO checklist (or similar), protocols and procedures.

9 Unclear or failed communication. Communication that should have been given wasn't or was inadequate or was misunderstood and not corrected.

10 Poor coordination. Failure in coordinating team activities; failure to anticipate the needs of the lead surgeon or lead anesthetist (surgeon at the console in robotic surgery).

11 Poor decision making. Failure to consider, select and communicate options; inadequacy or delay in implementing and reviewing decisions.

12 Poor situation awareness. Failure to gather and/or to integrate information or failure to use information to anticipate future tasks, problems and states of the operation.

13a Lack of experience of surgical team colleagues. Lack of experience of within surgical team, with the surgical procedure or technology.

13b Lack of experience of anesthetics team colleagues. Lack of experience of within anesthetic team, with the anesthetic procedure or technology

\begin{tabular}{cl}
\hline 14 & Fatigue. Mental fatigue or physical fatigue. \\
\hline 15 & Time pressure. Psychological stress resulting from experiencing a need \\
to get things done in less time than is required or desired.
\end{tabular}

16 Perioperative Emotional Stress. Stress induced by factors not directly related to the team, or to the characteristics and evolution of the intervention, e.g. responsibility for the budget and for other hospital objectives, organizational problems of the department, other critical patients, lawsuits.

17 Poor leadership. Failure to set and maintain standards or to support others in coping with pressure.

18 Team member familiarity. Team members unfamiliar with each other and each other's competencies.

19 Poor use of technology. Lack of ability to use relevant technology.

20 Inadequate ergonomics of equipment and work place. Equipment and workplace not designed to optimize usability and reduce operator fatigue and discomfort 
Table 2. Characteristics of respondents

\begin{tabular}{l}
\hline Number of respondents: 93 \\
\hline Age of respondents: from 29 to 81 \\
\hline Years of experience: from 1 to 52 \\
\hline Total number of surgical interventions: from 10 to 20000 \\
\hline Surgical setting: \\
Open surgery: $60 \%$ \\
Laparoscopic surgery: $40 \%$ \\
\hline
\end{tabular}

To investigate if the perceived influence of the IFs is significantly different under different surgical contexts (open vs laparoscopic surgery), Mann-Whitney Wilcoxon test (nonparametric test) was used. The difference of the perceived influence in the two surgical contexts resulted significant (threshold of significance, $p<0.05$ ) for five IFs: i) verbal interruptions $(\mathrm{p}=0.0210)$; ii) rude talk and disrespectful behaviors $(\mathrm{p}=0.0339)$; iii) unclear or failed communication $(\mathrm{p}=0.0290)$; iv) poor coordination $(\mathrm{p}=0.0385) ; \mathrm{v})$ poor situation awareness $(\mathrm{p}=0.0086)$. The descriptive statistics are reported in Figure 2, which presents the number of the respondents (obs), mean ratings, standard deviation, the minimum and maximum values in open and laparoscopic surgery. The descriptive profiles of the two study groups are also shown through box plots representation (Figure 3).

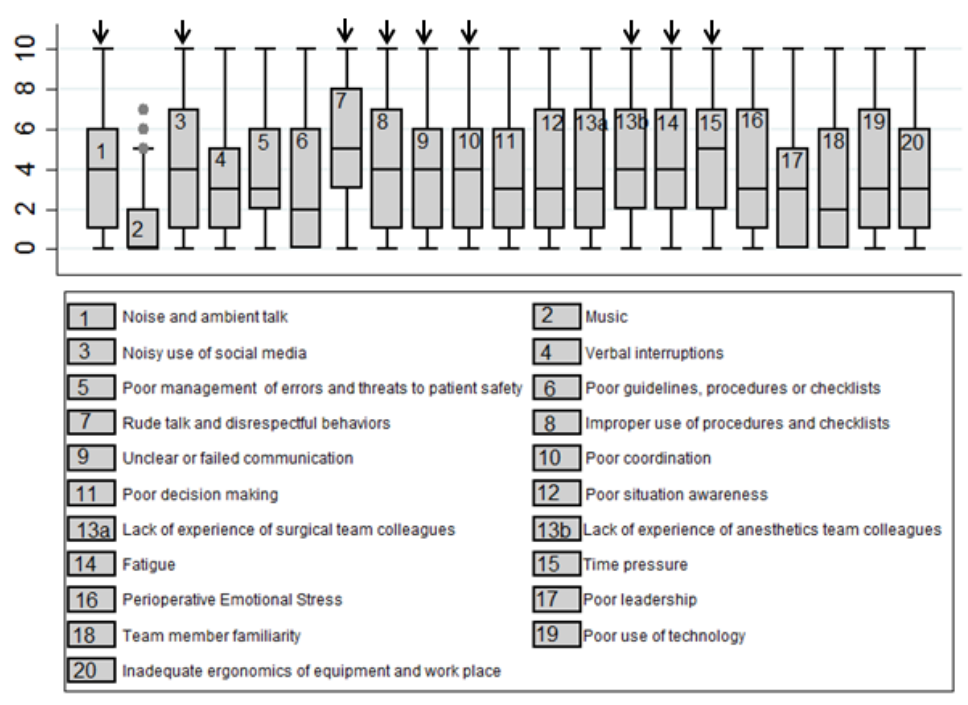

Fig. 1. Descriptive profiles of the perceived influence of each IF in a generic surgical setting (unique sample: 93 responses). 


\begin{tabular}{|crrrrr|}
\hline Open surgery & Obs & Mean & Std. Dev. & Min & Max \\
\cline { 2 - 6 } Verbal interruptions & 56 & 3 & 2.522625 & 0 & 9 \\
Rude talk and disrespectful behaviors & 56 & 4.660714 & 3.41801 & 0 & 10 \\
Unclear or failed communication & 56 & 3.446429 & 2.853512 & 0 & 10 \\
Poor coordination & 56 & 3.392857 & 3.049164 & 0 & 10 \\
Poor situation awareness & 56 & 3.232143 & 3.056981 & 0 & 10 \\
\hline Laparoscopic surgery & Obs & Mean & Std. Dev. & Min & Max \\
\cline { 2 - 6 } Verbal interruptions & 37 & 4.297297 & 2.644332 & 0 & 10 \\
Rude talk and disrespectful behaviors & 37 & 6.189189 & 2.923448 & 0 & 10 \\
Unclear or failed communication & 37 & 4.945946 & 3.299377 & 0 & 10 \\
Poor coordination & 37 & 4.864865 & 3.425088 & 0 & 10 \\
Poor situation awareness & 36 & 5.055556 & 3.430489 & 0 & 10 \\
\hline
\end{tabular}

Fig. 2. Descriptive statistics about the perceived influence of IFs resulted significantly different in the two surgical contexts.

Open surgery
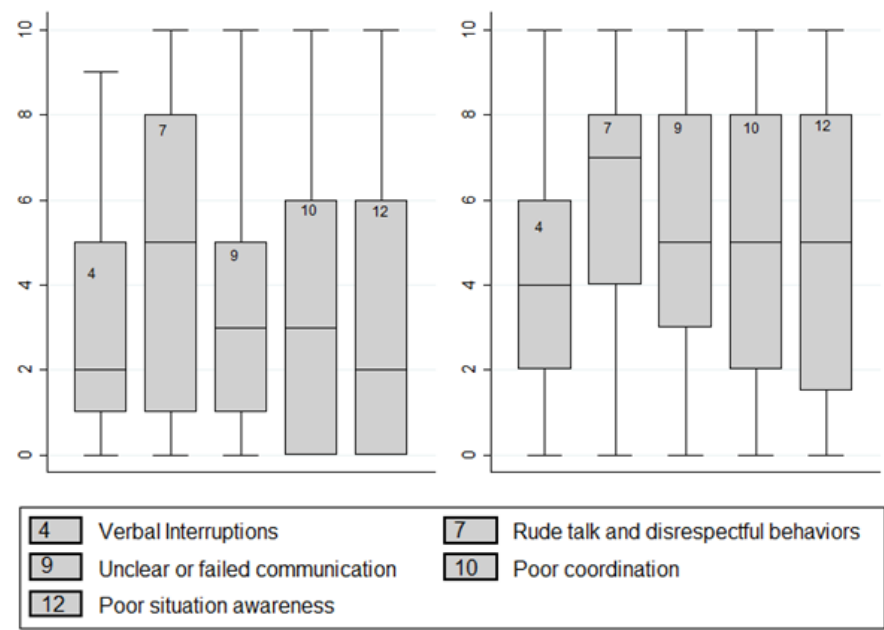

Fig. 3. Descriptive profiles of the perceived influence of IFs resulted significantly different in the two surgical context (the numbers refer to the corresponding number of the IFx shown in the table 1).

Surgeons perceive the influence of these five IFs as higher in laparoscopic surgery than in open surgery. Despite the maximum and minimum values similar (with the exception of IF 4, namely 'verbal interruptions', where the maximum value is higher for the laparoscopic setting), the medians are dramatically different: values in laparoscopic surgery are higher (either by 2 or 3 points) than in open surgery. In particular 'rude talk and disrespectful behaviors' and 'poor situation awareness' are those factors whose perceived influence present the medians 3 point higher in laparoscopic surgery than in open surgery.

Data clearly shows a strong influence of relational and communication issues on surgeon's performance (and surgical outcome as a consequence) under laparoscopic settings. These factors are perceived less influential by surgeons when referred to an open surgery context. 


\section{Conclusion}

The study represents the first step towards a quantitative analysis of human and organizational factors affecting surgeon's performance, surgical outcome and patient safety.

Providing a comprehensive taxonomy of influencing factors and relative perceived influence on surgeons' performance is both theoretically and managerially relevant. Theoretically, the study contributes to the validation of a taxonomy of IFs specifically designed for the healthcare sector, and surgery in particular. Moreover, to the best of authors' knowledge, the study is also the first documented attempt to estimate the strength of the influence (weight) of IFs on surgeon's performance; in this regard, the results of the study foster the application of quantitative Human Reliability Analysis (HRA) in surgery, which is an emerging research stream in patient safety literature. Finally, further research effort can be directed towards a better understanding of the underlying cognitive mechanisms that make the laparoscopic setting (or MIS in general) more vulnerable to poor relational and communication factors. Further research is also needed to expand the evidence base to the identification and assessment of the relevant IFs for robotic surgery.

The results of the present study can be also a useful reference for designing and implementing effective actions targeting patient safety improvements in surgery. Indeed, by focusing on the most influential human and organisational factors in different surgical contexts, risk managers can provide useful and precise inputs to organizational development, surgical training and other safety interventions programs.

\section{References}

[1] P. Joice, G. B. Hanna, and a Cuschieri, "Errors enacted during endoscopic surgery--a human reliability analysis.," Appl. Ergon., vol. 29, no. 6, pp. 40914, Dec. 1998.

[2] L. Chadwick and E. F. Fallon, "Human reliability assessment of a critical nursing task in a radiotherapy treatment process.," Appl. Ergon., vol. 43, no. 1, pp. 89-97, Jan. 2012.

[3] J. Ward, Y. Teng, T. Horberry, and P. J. Clarkson, "HEALTHCARE HUMAN RELIABILITY ANALYSIS - BY HEART," vol. 38, pp. 287-288, 2004.

[4] B. Tang, G. B. Hanna, N. M. a Bax, and a Cuschieri, "Analysis of technical surgical errors during initial experience of laparoscopic pyloromyotomy by a group of Dutch pediatric surgeons.," Surg. Endosc., vol. 18, no. 12, pp. 171620, Dec. 2004.

[5] A. D. Swain and H. E. Guttmann, "Handbook of Human Reliability Analysis with Emphasis on Nuclear Power Plant Applications Final Report," no. August, 1983.

[6] E. Hollnagel, Cognitive Reliability and Error Analysis Method (CREAM). 
Elsevier, 1998.

[7] J. C. Williams, HEART - A proposed method for achieving high reliability in process operation by means of human factors engineering technology in Proceedings of a Symposium on the Achievement of Reliability in Operating Plant, Safety and Reliability Society (SaRS). NEC, Birmingham, 1985.

[8] R. L. Boring, "How Many Performance Shaping Factors are Necessary for Human Reliability Analysis ?,” 2010.

[9] A. Kolaczkowski, J. Forester, E. Lois, and S. Cooper, "Good Practices for Implementing Human Reliability Analysis (HRA) (NUREG-1792)," Nureg1792, 2005.

[10] R. L. Boring and H. S. Blackman, "The origins of the SPAR-H method's performance shaping factor multipliers," 2007 IEEE 8th Hum. Factors Power Plants HPRCT 13th Annu. Meet., pp. 177-184, Aug. 2007.

[11] M. J. Barnes, D. Bley, and S. Cooper, "Technical basis and implementation guidelines for a technique for human event analysis (ATHEANA)," NUREG1624, Rev, 2000.

[12] J. W. Kim and W. Jung, "A taxonomy of performance influencing factors for human reliability analysis of emergency tasks," J. Loss Prev. Process Ind., vol. 16, no. 6, pp. 479-495, Nov. 2003.

[13] R. Onofrio, P. Trucco, and A. Torchio, "Towards a Taxonomy of Influencing Factors for Human Reliability Analysis (HRA) Applications in Surgery," Procedia Manuf., vol. 3, pp. 144-151, 2015.

[14] R. a Krueger and M. A. Casey, "Focus groups: A practical guide for applied research," Rev. Lit. Arts Am., vol. 22, pp. 129-152, 2000. 